\title{
Reviewer Acknowledgments
}

The editors gratefully acknowledge the generous assistance of the many excellent reviewers who served AMBIO in 2014 .

Per Arild Aarrestad

Juan Acosta

Terando Adam

Vanessa Adams

Wisdom Akpalu

Zeshan Ali

Jukka Alm

Antonio Almeida

Mark Altaweel

Hans Andersen

Leif Anderson*

Erik Andersson**

Agneta Andersson

Margaret Andrew

Steven R. Archer

Jonas Ardö

Manuel Arias-Estévez

Frederick Ato Armah

Derek Armitage

Ayyanadar Arunachalam

Antonia Dolores Asencio

Engdawork Assefa

Xuemei Bai

Timothy Baird

Martha Bakker

Kunshan Bao

Åke Barklund

Agustina Barros

Richard Batiuk

Seth Baum

Kamal Bawa

José Benatti

Andrew Bengsen
Håkan Berg
Per Berggren
Sten Bergström
Charlotte Berkström
Charles Besancon
Gilles Billen
Asit Biswas
Andrea Lucia Biswas Tortajada
Mats Björk
Kristina Blennow
Wiebren Johannes Boonstra
Kevin J. Boyle
Arvid Bring
Andrea J. Britton
Sara Brogaard
Peter Bruce
Ishi Buffam
Bård-Jørgen Bårdsen
Ingeborg Callesen
Pep Canadell
Jesus Carrera
Kenneth Cassman
Timothy T. Caughlin
Jonas Cedergren
Terry Chapin
Anna Christiernsson
Miguelito Clavero
Dennis Collentine
Drew Conin
Daniel Conley**
Florence Conteh
Aration

Andrew Bengsen

Håkan Berg

Per Berggren

Sten Bergström

Charlotte Berkström

Charles Besancon

Gilles Billen

Asit Biswas

Andrea Lucia Biswas Tortajada

Mats Björk

Kristina Blennow

Wiebren Johannes Boonstra

Kevin J. Boyle

Arvid Bring

Andrea J. Britton

Sara Brogaard

Peter Bruce

Ishi Buffam

Bård-Jørgen Bårdsen

Ingeborg Callesen

Kenneth Cassman

Timothy T. Caughlin

Jonas Cedergren

Terry Chapin

Anna Christiernsson

Miguelito Clavero

ennis Coll

Daniel Conley**

(C) Royal Swedish Academy of Sciences 2015 www.kva.se/en

\author{
Perran Cook \\ Claudia Cordovil \\ Michael Cox \\ Stockwell Craig \\ Bogdan Cristescu \\ Graeme Cumming \\ Andre Cunha \\ Vladimir Cvetkovic \\ Miikka Dal Maso \\ Lars Dalby \\ Åsa Danielsson \\ Lisa Darby \\ Bijan Dargahi \\ Mark David \\ Mark Davis \\ Fabrice DeClerck \\ Narayan Prasad Dhital \\ Amy Diedrich \\ Peter Dorward \\ Michael R. Dove \\ Hari Dulal \\ Jennifer Dyer \\ Anthony C. Edwards \\ Anneli Ekblom \\ Libor Ekrt \\ David Eldridge \\ Chris S. Elphick \\ Hampus Eriksson \\ Hillevi Eriksson \\ Getachew Tadesse Eshete \\ Louisa Evans \\ Eugene Ezebilo
}


Christo Fabricius

Malin Falkenmark

Aulia Riza Farhan

David Fazzino

Rasmus Fensholt

David Fernández-Calviño

Maria José Fernández-Sanjurjo

Cristian-Alarcon Ferrari

Brian G. Fitzgerald

Carl Folke*

Maureen Fordham

Peter Fredman

Nikolai Friberg

Matt Frost

Guanglei Gao

Karin Gerhardt

Jan Ove Gjershaug

Michael Goodsite

David Granlund

Andy Green

Nancy Grimm

Jolanta Grochowskaja

R. Edward Grumbine

Annemieke Gärdenäs

Nomana Intekhab Hadi

Karl Havens

Mads Peter Heide-Jørgensen

Stefanie Herrmann

Benjamin Heumann

Kristina Hill

Stephanie Hitztaler

Ken Hodgkinson

Meg Holden

George Holmes

Grete K. Hovelsrud

David Hubbard

Christoph Humborg

Hans Hurni*

Kristoffer Hylander

Johan Höjesjö

Greger Hörnberg

Ulrik Ilstedt

Fredrik Ingemarson

Yoshio Inoue

Mine Islar

Sverker Jagers

Neli Shalva Jamaspashvili

Jerker Jarsjo

James Jawitz

Henning Jensen

Martin Rudbeck Jepsen

Arne Jernelöv**
Janice Jiggins

Kerstin Johannesson

Margareta Johansson

Johanna Johnson

Julie Johnson

Jörgen Johnsson

Alan Jones

Thomas Jordan

Wolfgang Junk

John Juston

Pavel Kabat

Bjorn Kaltenborn

Markku Kanninen

Ryan P. Kelly

Carina Keskitalo**

Ahmed Khan

Holger Kirchmann

Otto Klemm

Patrik Klintenberg

Torsten Krause

Jakub Kronenberg

Timo Kuuluvainen

Ella-Maria Kyrö

Thomas Lacher, Jr.

Lars Laestadius

Per Lagerås

Glenn-Marie Lange

Paul Laris

Hjalmar Laudon

Sandra Lavorel

Joseph H.W. Lee

Katrianne Lehtipalo

Gun Lidestav

Mats Lindegarth

John Linnel

Kunza Lisa

Yansui Liu

Barbara Livoreil

Elisa Maria Lopez

Torbjörn Lundh

Steve W. Lyon

Tomas Lämås

Edward Mabaya

Teresa Magro

Anders Malmer**

Mariette Manktelow

John Marchant

Jochen Markard

Melissa Marschke

Helene Marsh

Paul Marshall

Nadine Anne Marshall
Maria Jose Martinerz-Harmsa

Antonio Maturo

Anastasios Mavrakis

Jim McLaughlin

Elizabeth McLeod

Jeffrey McNeely*

Robert Mendelsohn

Roel Merckx

Juha Merilä

Chiyuan Miao

Grzegorz Mikusiński

John Miringa

Malin Mobjörk

Pablo Modernel

Khola Mogotsi

Philippe Moguedet

Sylvie Morardet

Ignacio Morell

Carl-Magnus Morth

Inger Elisabeth Måren

David C. Natcher

Y.Y. Neo

Barry Ness

David Newsome

Emmanuel Njukwe

Anna Nordén

Tomasz Okruszko

Gunilla A. Olsson

Madelene Ostwald

Jane Packard

Matilda Palm

Gisli Palsson

Manuela Panzacchi

Jyothi Parikh

Fernanado C. Passos

Frederick Payton

Eliza Pennypacker

Lars Persson

Lars Pettersson

Alessandro Pezzoli

Jana Pickova

Louisa Ponnampalam

Gil Pontius

Neil Powell

Stephen Prince

Changbo Qin

Emmanuelle Quillerou

Lars Rahm

Asad Rahmani

Rajesh Kumar Rai

Vasna Ramasar

Carleton Ray 
Maureen G. Reed

Ilona Riipinen**

Jesper Riis Christiansen

Claudia Ringler

Roseline Roseline Remans

Ed Rowe

Graciela M. Rusch**

Murray Rutherford

Prasanna Samal

Mats Sandewall

Leonard Sandin

Fred P. Saunders

Robert S. Schemenauer

Marianne Schmink

Michael Schoon

Lisen Schultz

Chris Searcy

Rahul Shrivastava

Jonathan Silver

Javier Simonetti

Martin Skov

Matthew Slater

Caroline Slomp

Carsten Smith-Hall

Ekaterina Sokolova
Sabrina Spatari

Jesper Stage**

Edward Stets

Carly Stevens

Anders Stigebrandt

Per Stålnacke

Cecilia Sundberg

Melinda Sundell

Mark Swilling

Martin Sykes

Ivo Syndicus

Péter Szabó

Alan Tappin

Michael Tedengren**

David Tenenbaum

Vernon George Thomas

Hale W. Thurston

Pernilla Tidåker

Renae Tobin

Hiroto Toda

Lars Tranvik**

Hans Tømmervik

Erik Valinger

Mohammad Valipour

René van der Wal
Paule Vasseur

Louis Verchot

Niels Vestergaard

Alexei Voinov

Xiuhong Wang

Yafeng Wang

Gao Wangsheng

Kara Webster

Da Wei

Adrian Werner

Kerstin Westin

Bradford Wilcox

Richard Wright

Angela Wulff**

$\mathrm{Yu} \mathrm{Ye}$

Alexander J. B. Zehnder*

Jianjun Zhou

Davide Zilli

Francisco Zorondo

Lars Östlund

* serves in AMBIO's Editorial Board

** serves as Associate Editor for AMBIO 